\title{
IMPORTÂNCIA DAS FLORESTAS E PRINCIPAIS FONTES DE INFORMAÇÃO PARA AGRICULTORES DO NORTE DO RIO GRANDE DO SUL
}

\author{
Cleusa Vicente Vargas ${ }^{1}$ \\ Sônia Beatris Balvedi Zakrzevski²
}

Resumo: O estudo teve por objetivo identificar e caracterizar as percepções de agricultores, sobre a importância das florestas, para as atividades agropecuárias. Também buscou verificar se as fontes de informação e de formação sobre o tema interferem nas suas percepções. Os dados foram obtidos por meio de entrevista e submetidos à análise de conteúdo e à análise estatística. A pesquisa evidenciou que os agricultores reconhecem inúmeros serviços ecossistêmicos, prestados pelas florestas, com destaque aos serviços de regulação. $O$ tipo de agricultura praticada e as fontes de informação utilizadas são fatores que interferem nas percepções, dos agricultores pesquisados, sobre as florestas.

Palavras-chave: Percepções; Serviços Ecossistêmicos; Educação Ambiental; Sustentabilidade; Ecologia.

\begin{abstract}
The study aimed to identify and characterize the perceptions of farmers, about the importance of forests, for agricultural activities. It also sought to verify whether the sources of information and training on the topic interfere with their perceptions. The data were obtained through interviews and submitted to content analysis and statistical analysis. The research showed that farmers recognize innumerable ecosystem services, provided by forests, with emphasis on regulation services. The type of agriculture practiced and the sources of information used are factors that interfere in the perceptions of the surveyed farmers about the forests.
\end{abstract}

Keywords: Perceptions; Ecosystem Services; Environmental Education; Sustainability; Ecology.

\footnotetext{
1 Universidade Regional Integrada do Alto Uruguai e das Missões, Erechim, RS.

E-mail: cleusavvargas@hotmail.com. Link para o Lattes: http://lattes.cnpq.br/3312075440493395 2Universidade Regional Integrada do Alto Uruguai e das Missões, Erechim, RS.

E-mail: sbz@uricer.edu.br. Link para o Lattes: http://lattes.cnpq.br/3829730130976408
}

Revbea, São Paulo, V. 15, № 7: 259-276, 2020.

revista brasileira educação ambiental 


\section{Introdução}

Entre os ecossistemas terrestres, as florestas se destacam pela exuberância, biodiversidade e pela capacidade de fornecer uma gama de serviços ecossistêmicos fundamentais para a sobrevivência de muitas espécies, além de suprirem as necessidades humanas (CHAO, 2012; GAMFELDT et al., 2013). Aproximadamente $25 \%$ da população mundial dependem diretamente das florestas para viver (ONU, 2015) - eles contribuem no combate à pobreza rural; garantem a segurança alimentar e proporcionam às pessoas meios de subsistência e, também, fornecem inúmeros serviços ambientais vitais (FAO, 2018).

As florestas estão entre os mais importantes repositórios de biodiversidade - as florestas tropicais sozinhas hospedam, pelo menos, dois terços das espécies terrestres (GARDNER et al., 2009). E o seu manejo sustentável é essencial não apenas para a conservação, mas também para sustentar o funcionamento do ecossistema e, assim, o fornecimento contínuo e saudável de inúmeros serviços ecossistêmicos, como a produção de alimentos. A literatura apresenta inúmeros serviços ecossistêmicos por elas prestados, com destaque para a oferta e a disponibilidade de água, à regulação dos microclimas, à formação do solo, à produção de madeira, fibras, fármacos e alimentos, além da função de informação e bem-estar espiritual (VARJABEDIAN, 2010; DE SOUSA et al., 2017). Também é apontada a importância das florestas na redução dos desastres naturais como secas, enchentes, deslizamentos de terra, assoreamento de corpos-d'água, dentre outros eventos (NEDEL; SAUSEN; SAITO, 2012; FINOTTI; SANTOS, 2013; SINARE; GORDON; KAUTSKY, 2016). E, em nível global, as florestas, por meio do sequestro de carbono, mitigam as mudanças climáticas; atuam no equilíbrio de dióxido de carbono e de oxigênio; umidade no ar, além de servir de proteção para os recursos hídricos de todo o Planeta (UNITED NATIONS, 2015).

Nosso Planeta possui uma área de 4,06 bilhões de hectares (ha), ou seja, $31 \%$ da área total, cobertos por florestas. Os biomas tropicais têm a maior proporção de florestas do mundo (45\%), seguidos pelos domínios boreal, temperado e subtropical. Porém, $54 \%$ das florestas globais estão em, apenas, cinco países - Federação Russa, Brasil, Canadá, Estados Unidos da América e China. E a América do Sul tem a maior parcela de florestas em áreas protegidas, com $31 \%$ (FAO, 2020).

No entanto, os ecossistemas florestais vêm sendo intensamente devastados pela exploração madeireira; pela introdução de espécies exóticas; pela urbanização (principalmente em regiões litorâneas); pelas atividades de mineração; pelos empreendimentos hidrelétricos, pela expansão da agricultura e da pecuária, dentre outros. No Brasil, a agricultura convencional é apontada, pela literatura, como um dos grandes responsáveis pelas perdas de florestas, comprometendo o equilíbrio dos ecossistemas e a saúde das populações (CAMPANILI; SCHÄFFER, 2010; SOARES-FILHO et al., 2014; FRASER et al., 
2016). De outro lado, os sistemas agroecológicos de produção têm se mostrado uma importante estratégia para a conservação dos recursos naturais e da biodiversidade (SILVA, 2016), incluindo aspectos ecológicos, econômicos e sociopolíticos (BEGON; TOWNSEND; HARPER, 2007). Suas estratégias estão associadas à conservação das comunidades biológicas e da diversidade genética mediante a manutenção da paisagem, valorizando as espécies nativas e reduzindo as pressões sobre os ecossistemas (PERFECTO; VANDERMEER, 2008).

A conservação das florestas depende de políticas públicas, mas principalmente, da ação direta dos seres humanos nos ecossistemas a partir do entendimento, destes, quanto à sua importância (ZAKRZEVSKI, VARGAS, DECIAN, 2020). Os diferentes olhares determinam a relação que o indivíduo tem com o ambiente e compreendê-los é o primeiro passo para discussão, adoção, ampliação e/ou redirecionamentos de estratégias de proteção e intervenção, na perspectiva de amenizar os impactos e as perdas de florestas.

Com relação às florestas, na última década inúmeros estudos foram desenvolvidos com o intuito de compreender as percepções, dos agentes de transformação, dos ecossistemas naturais e de compreender a amplitude dos problemas ambientais, bem como de construir soluções de uso sustentável das florestas, mantendo a sua biodiversidade e garantindo o fornecimento dos serviços ecossistêmicos (QUINTAS-SORIANO et al., 2018; MEIJAARD et al., 2013; OUKO et al., 2018; FRICK; BAUER; LINDERN; HUNZIKER, 2018; ZAKRZEVSKI; VARGAS; DECIAN, 2020), identificando a influência de fatores sociais, econômicos, culturais na relação das pessoas com as florestas. Esses estudos apontam que a compreensão dos conceitos e dos valores, que permeiam as ações dos agricultores e sua relação com as áreas de florestas, contribui para subsidiar o planejamento e a elaboração de programas educativos voltados à adoção de práticas dirigidas à conservação da biodiversidade, favorecendo propostas que considerem as necessidades locais e possibilitem o engajamento da comunidade.

Este estudo tem por objetivo identificar e caracterizar as percepções de produtores rurais, convencionais e agroecológicos, residentes no Norte do RS, sobre a importância das florestas para as atividades agropecuárias. Além disso, verificar se as fontes de informação e de formação dos agricultores sobre o tema influenciam as suas percepções.

\section{Metodologia}

A pesquisa foi desenvolvida no Norte do Rio Grande do Sul (RS), Sul do Brasil, abrangendo duas Regiões Funcionais de Desenvolvimento (Corede Norte e Corede Celeiro), situadas no território pertencente ao bioma Mata Atlântica (Figura 1). 


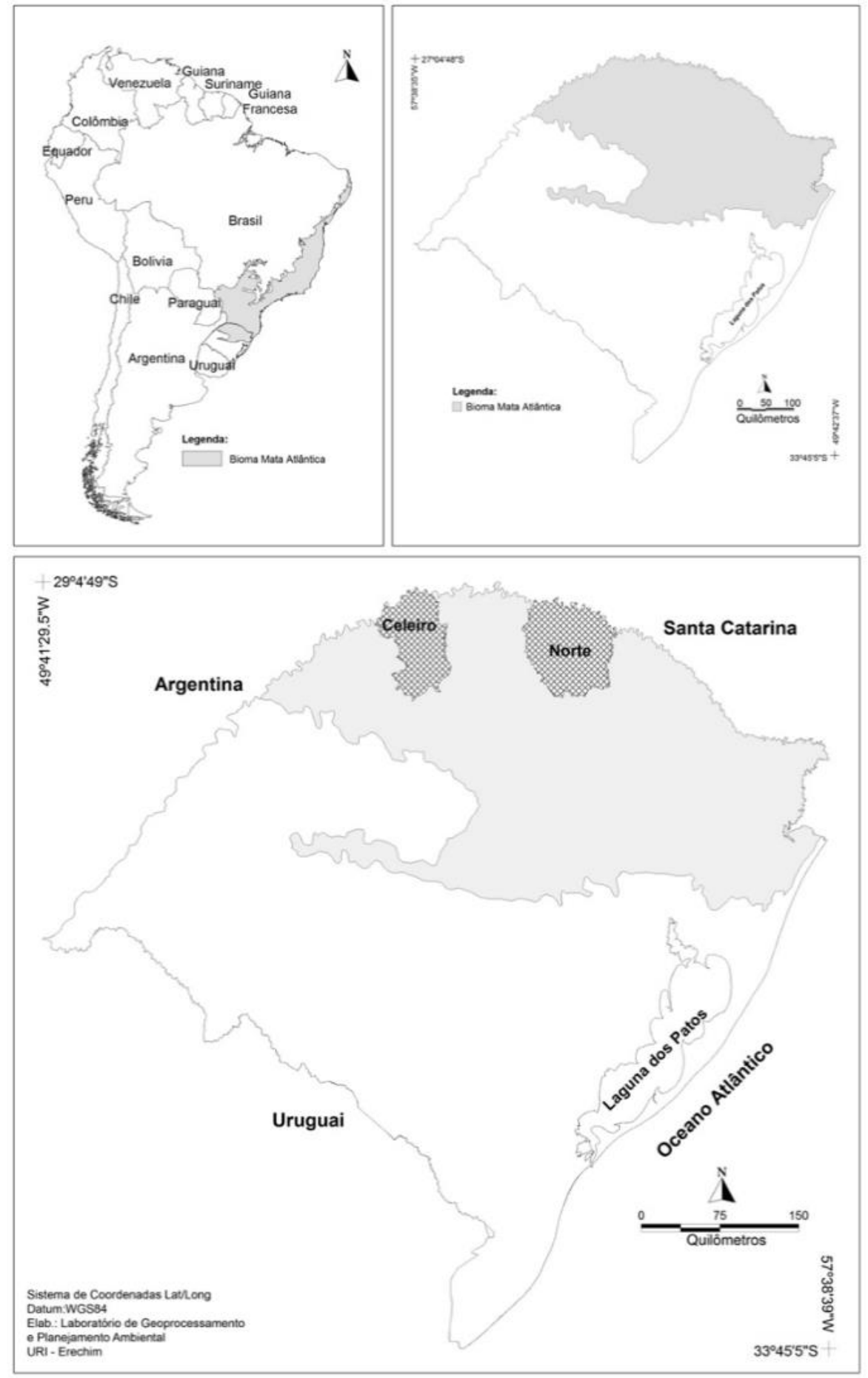

Figura 1: Localização das áreas de estudo - Corede Norte e Corede Celeiro.

Fonte: Lageplam (URI, 2018).

O RS tem a agropecuária como um dos setores mais importantes, com destaque para a agricultura que representa, aproximadamente, $10 \%$ do PIB brasileiro (FEIX; LEUSIN, 2015). A agricultura familiar é responsável por $30 \%$ da produção, ocupando $86 \%$ dos estabelecimentos agrícolas gaúchos, sendo que $38,9 \%$ dos estabelecimentos agrícolas são menores que 10 ha e, apenas, $2 \%$ maiores que 500 ha (RS, 2014). O Estado abriga 13,4\% dos 
estabelecimentos orgânicos certificados do País, e houve um aumento de $342,59 \%$ no número de estabelecimentos, certificados no Estado, se comparados os dados atuais com o Censo Agropecuário de 2006 (IBGE, 2017).

O Norte do Estado caracteriza-se por uma forte tradição na atividade agrícola diversificada, voltada à produção de grãos - soja, milho e trigo e à pecuária, com a produção de leite, criação de aves e suínos. Caracteriza-se pela presença de pequenas propriedades e com a utilização de obra familiar (RS, 2017). A Região, originariamente, apresentava a maior área de florestas do Estado. Era coberta por fisionomias naturais da Floresta Ombrófila Mista e da Floresta Estacional Decidual. Atualmente, sua paisagem encontra-se fragmentada, sendo dominada por fisionomias agrícolas (CORDEIRO; HEINRICH, 2009). Os fragmentos melhor conservados estão localizados em áreas bastante íngremes, impróprias para a mecanização agrícola.

Participaram do estudo 120 agricultores, amostrados entre os produtores convencionais e agroecológicos, a partir de listagens disponibilizadas pelas Secretarias Municipais de Agricultura. De cada Corede, participaram: i) 30 produtores convencionais, ou seja, os que manejam sistemas de produção agrícola modernos, em que predominam as técnicas intensivas, pelo uso de insumos e tecnologias; ii) 30 produtores agroecológicos, ou seja, os que manejam sistemas agrícolas de base ecológica, praticando uma agricultura mais próxima da agricultura tradicional (Figura 2).

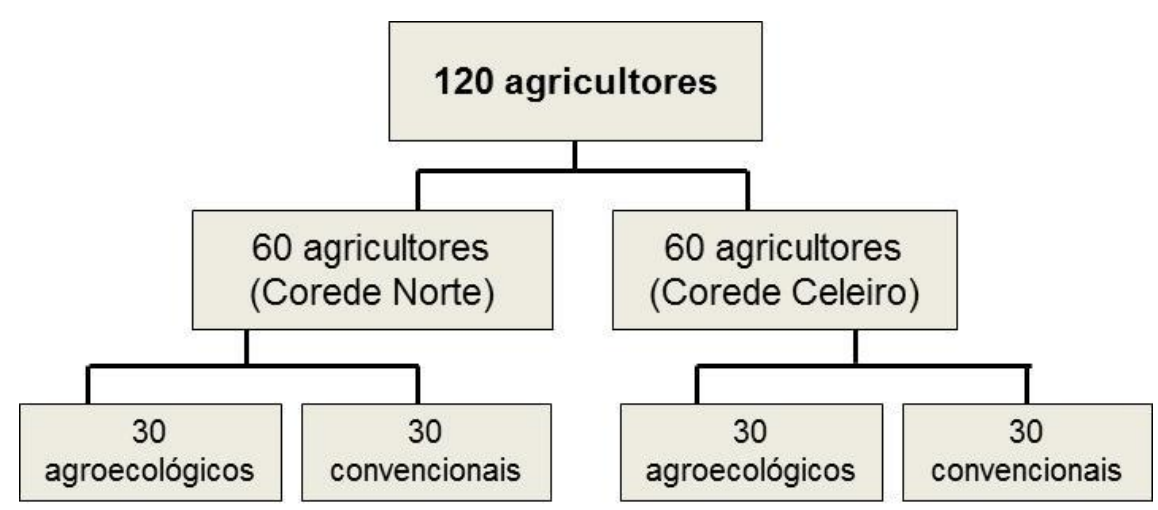

Figura 2: Participantes da pesquisa

Fonte: Compilado pelos autores a partir dos dados primários (2019).

Dos participantes da pesquisa, 66,66 \% são do gênero masculino; a maioria (75\%) reside no meio rural e tem idade entre 40 e 59 anos $(56,66 \%)$. Em relação ao nível de instrução, 42,83\% têm Ensino Fundamental; 29,16\%, Ensino Médio, e 25\%, Ensino Superior (Tabela 1). Os agricultores agroecológicos e familiares têm, nas cerealíferas, o principal cultivo, mas também praticam a fruticultura, silvicultura, horticultura, a produção de bulbos e raízes. Já os agricultores convencionais e não-familiares têm a produção baseada nos monocultivos, na pecuária de corte e na de leite. 
Tabela 1: Caracterização dos agricultores, residentes no Norte do RS, participantes da pesquisa, agrupados, segundo o tipo de agricultura praticada na propriedade; sexo, idade e escolaridade.

\begin{tabular}{llc}
\hline & VARIÁVEIS & N. de participantes \\
\hline Tipo de & Agroecológicos & 60 \\
Agricultura & Convencionais & 60 \\
\hline Sexo & Feminino & 40 \\
& Masculino & 80 \\
\hline \multirow{2}{*}{ Idade } & 20 a 39 anos & 25 \\
& 40 a 59 anos & 68 \\
& 60 anos ou mais & 27 \\
\hline Escolaridade & Ensino Fundamental & 55 \\
& Ensino Médio & 35 \\
& Ensino Superior & 30 \\
\hline
\end{tabular}

Fonte: Compilado pelos autores a partir dos dados primários (2019).

A coleta de dados foi realizada no período de fevereiro de 2017 a março de 2018, por meio de entrevista individual, aplicada em data, local e horário, definidos com cada participante do estudo, após a aprovação do projeto pelo Comitê de Ética em Pesquisa, da URI - Erechim (Parecer 2.452.137/2017), e após a autorização dos participantes, por meio da assinatura de um Termo de Consentimento Livre e Esclarecido. O instrumento de pesquisa foi constituído por questões abertas, com informações para: caracterização socioeconômica e cultural dos entrevistados; identificação das percepções sobre a importância das florestas para a agropecuária; identificação das fontes de informações acessadas, estratégias de formação e entidades/empresas/órgãos de assistência que orientam as suas ações. As informações, obtidas durante as entrevistas, foram registradas em um formulário elaborado pela pesquisadora e, também, gravadas, em meio digital, com prévia autorização dos participantes. Após, os dados de cada pergunta foram submetidos a um processo de análise de conteúdo (BARDIN, 1977). O conjunto de dados de cada questão foi organizado em planilhas, no Microsoft Excel, e submetido a um processo de análise descritiva, com o objetivo de evidenciar as características de distribuição das variáveis. Os dados também foram submetidos ao teste do qui-quadrado $\left(x^{2}\right)$ com $\mathrm{p}<0,05$, buscando verificar se o tipo de agricultura praticada, as fontes de informação e de formação recebidas interferem nas percepções dos agricultores sobre a importância das florestas. As análises foram realizadas, utilizando-se o software Bioestat 5.0. 


\section{Resultados e discussão}

\section{Importância das florestas para a atividade agropecuária}

O estudo identificou que a maioria dos agricultores pesquisados $(81,66 \%)$ reconhece que as florestas são importantes para a agricultura. Nesse aspecto, há uma diferença significativa entre os produtores agroecológicos e os convencionais $\left(x^{2}=2693 ; \mathrm{gl}=1 ; \mathrm{p}<0.0001\right)$. Também se observou a relação entre a importância das florestas e a idade dos participantes: quanto maior a idade, menor é a percepção da importância das florestas para as atividades agropecuárias (Figura 3)

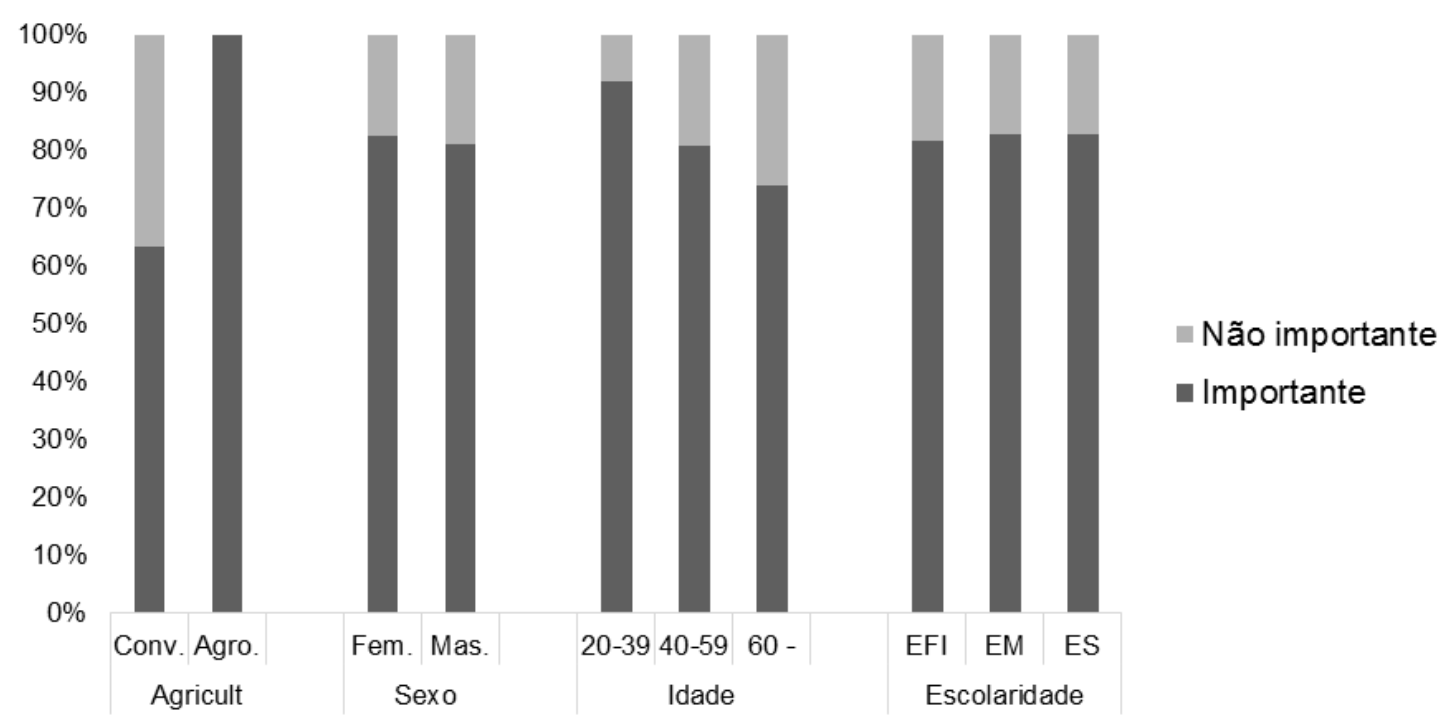

Figura 3: Importância atribuída às florestas para a agricultura (\%), segundo produtores rurais, residentes no Norte do RS, agrupados segundo o tipo de agricultura praticada na propriedade; sexo, idade e escolaridade.

Fonte: Compilado pelos autores a partir dos dados primários (2019).

É muito provável que $100 \%$ dos produtores agroecológicos reconheçam a importância das florestas, em função do sistema de produção adotado, que tem, como princípios, a preservação da biodiversidade, dos ecossistemas e do patrimônio genético. Os agricultores buscam produzir alimentos saudáveis, livres de transgênicos e de agrotóxicos, tendo como referência os saberes e as culturas dos povos do campo, conservando as águas e as florestas e defendendo a vida.

Por meio de 3.526 citações (média de 2,93 citações por agricultor), os agricultores listaram 8 serviços ecossistêmicos prestados pelas florestas, que, em suas percepções, contribuem para as atividades agropecuárias: as florestas contribuem na manutenção de temperaturas mais amenas $(50,83 \%)$; na manutenção da umidade do solo (49,16\%); na proteção das lavouras, servindo como quebra-vento $(49,16 \%)$; na manutenção de polinizadores $(34,16 \%)$; no controle de processos erosivos $(33,33 \%)$; no controle biológico, evitando 
pragas e doenças (33,33\%); na barreira contra poluentes e doenças $(29,16 \%)$; na ciclagem de nutrientes (14,16\%). Há uma diferença expressiva entre a média de citações de serviços entre os produtores agroecológicos e os convencionais - média de 1,38 serviços entre os convencionais e de 4,48 serviços entre os agroecológicos (Tabela 2). Essa diferença existe entre as porcentagens de citações em relação a todos os serviços prestados pelas florestas $\left(x^{2}=23,72 ; g l=9 ; p=0,05\right)$.

Também se evidencia que o nível de escolarização dos produtores e a idade são dois fatores que parecem influenciar as percepções dos serviços ecossistêmicos prestados pelas florestas - a média de citações de serviços aumenta conforme se eleva o nível de instrução e diminui a idade dos participantes. São os produtores mais jovens, e com maior instrução, que citam maior número de serviços. É importante ressaltar que esse dado é diferente daquele encontrado em outros estudos: Sodhi et al. (2010); Garcia, Fantini e Salvador (2016), Quintas-Soriano et al. (2018) verificaram que os agricultores, com mais idade, são os que citam maior número de serviços ecossistêmicos, se comparados aos mais jovens.

Tabela 2: Importância das florestas para as atividades agropecuárias, segundo produtores rurais, residentes no Norte do RS, agrupados segundo o tipo de agricultura praticada; sexo, idade e escolaridade (\% de citações).

\begin{tabular}{|c|c|c|c|c|c|c|c|c|c|c|}
\hline \multirow[b]{2}{*}{$\begin{array}{c}\text { Importância das } \\
\text { florestas }\end{array}$} & \multicolumn{2}{|c|}{ Agricultura } & \multicolumn{2}{|c|}{ Sexo } & \multicolumn{3}{|c|}{ Idade } & \multicolumn{3}{|c|}{ Escolaridade } \\
\hline & $\begin{array}{l}\text { Conv. } \\
\mathrm{n}=60\end{array}$ & $\begin{array}{l}\text { Agro. } \\
\mathrm{n}=60\end{array}$ & $\begin{array}{l}\text { Fem } \\
n=40\end{array}$ & $\begin{array}{l}\text { Masc } \\
\mathrm{n}=80\end{array}$ & $\begin{array}{l}20-39 \\
\mathrm{n}=25\end{array}$ & $\begin{array}{l}40-59 \\
\mathrm{n}=68\end{array}$ & $\begin{array}{l}+60 \\
\mathrm{n}=27\end{array}$ & $\begin{array}{c}E F \\
n=55\end{array}$ & $\begin{array}{c}\mathrm{EM} \\
\mathrm{n}=35\end{array}$ & $\begin{array}{c}E S \\
n=30\end{array}$ \\
\hline $\begin{array}{l}\text { Temperaturas mais } \\
\text { amenas }\end{array}$ & 40.0 & 61.7 & 47.5 & 52.5 & 64.0 & 58.8 & 18.5 & 41.8 & 48.6 & 66.7 \\
\hline $\begin{array}{l}\text { Servem como } \\
\text { quebra-vento }\end{array}$ & 26.7 & 71.7 & 50.0 & 48.8 & 48.0 & 54.4 & 37.0 & 54.5 & 45.7 & 43.3 \\
\hline $\begin{array}{l}\text { Mantêm a umidade } \\
\text { do solo }\end{array}$ & 30.0 & 68.3 & 55.0 & 46.3 & 40.0 & 54.4 & 44.4 & 47.3 & 45.7 & 56.7 \\
\hline Controlam a erosão & 20.0 & 46.7 & 30.0 & 35.0 & 40.0 & 35.3 & 22.2 & 38.2 & 25.7 & 33.3 \\
\hline $\begin{array}{l}\text { Ciclagem - } \\
\text { nutrientes }\end{array}$ & 1.7 & 26.7 & 12.5 & 15.0 & 28.0 & 11.8 & 7.4 & 5.5 & 5.7 & 40.0 \\
\hline $\begin{array}{l}\text { Barreira/poluentes/ } \\
\text { doenças }\end{array}$ & 6.7 & 51.7 & 40.0 & 23.8 & 60.0 & 20.6 & 22.2 & 23.6 & 28.6 & 40.0 \\
\hline $\begin{array}{l}\text { Mantêm } \\
\text { polinizadores }\end{array}$ & 8.3 & 60.0 & 45.0 & 28.8 & 44.0 & 36.8 & 18.5 & 23.6 & 42.9 & 43.3 \\
\hline $\begin{array}{l}\text { Controle biológico - } \\
\text { controle de pragas }\end{array}$ & 5.0 & 61.7 & 45.0 & 27.5 & 52.0 & 35.3 & 11.1 & 20.0 & 37.1 & 53.3 \\
\hline
\end{tabular}

Fonte: Compilado pelos autores a partir dos dados primários (2019). 
pela água, com as plantas cultivadas; ii) sombreamento das áreas cultivadas, diminuindo a produção; iii) restrição de áreas para produção em função da regularização ambiental prevista pela Lei de Proteção da Vegetação Nativa. Nesse aspecto, há diferenças entre os agricultores com diferentes idades $\left(x^{2}=28,57 ; \mathrm{gl}=2 ; \mathrm{p}<0,001\right)$; entre homens e mulheres $\left(x^{2}=16,81 ; \mathrm{gl}=2 ; \mathrm{p}<0,01\right)$; e também entre aqueles com diferentes níveis de instrução $\left(x^{2}=50,92 ; \mathrm{gl}=2\right.$; $\mathrm{p}<0,001$ ) (Tabela 3).

Tabela 3: Prejuízos gerados pela manutenção de florestas para as atividades agropecuárias (\%), segundo produtores rurais, residentes no Norte do RS, agrupados segundo o tipo de agricultura praticada; sexo, idade e escolaridade (\% de citações)

\begin{tabular}{lccccccccccc}
\hline \multirow{2}{*}{ Prejuízos } & \multicolumn{1}{c}{ Agricultura } & \multicolumn{2}{c}{ Sexo } & \multicolumn{3}{c}{ Idade } & \multicolumn{3}{c}{ Escolaridade } \\
\cline { 2 - 12 } & $\begin{array}{l}\text { Conv. } \\
\mathrm{n}=60\end{array}$ & $\begin{array}{c}\text { Agro. } \\
\mathrm{n}=60\end{array}$ & $\begin{array}{c}\text { Fem } \\
\mathrm{n}=40\end{array}$ & $\begin{array}{c}\text { Masc } \\
\mathrm{n}=80\end{array}$ & $\begin{array}{c}20-39 \\
\mathrm{n}=25\end{array}$ & $\begin{array}{c}40-59 \\
\mathrm{n}=68\end{array}$ & $\begin{array}{c}+60 \\
\mathrm{n}=27\end{array}$ & $\begin{array}{c}\mathrm{EF} \\
\mathrm{n}=55\end{array}$ & $\begin{array}{c}\mathrm{EM} \\
\mathrm{n}=35\end{array}$ & $\begin{array}{c}\mathrm{ES} \\
\mathrm{n}=30\end{array}$ \\
\hline $\begin{array}{l}\text { Redução áreas } \\
\text { de plantio }\end{array}$ & 11.7 & 0.0 & 2.5 & 7.5 & 16.0 & 1.5 & 7.4 & 5.5 & 2.9 & 10.0 \\
\hline $\begin{array}{l}\text { Ressecamento } \\
\text { do solo }\end{array}$ & 28.3 & 0.0 & 7.5 & 17.5 & 8.0 & 14.7 & 18.5 & $\begin{array}{r}18 . \\
2\end{array}$ & 11.4 & 10.0 \\
\hline $\begin{array}{l}\text { Sombreamento } \\
\text { das lavouras }\end{array}$ & 11.7 & 0.0 & 7.5 & 5.0 & 8.0 & 5.9 & 3.7 & 5.5 & 5.7 & 6.7 \\
\hline
\end{tabular}

Fonte: Compilado pelos autores a partir dos dados primários (2019).

Os produtores, em especial os agroecológicos, atribuem importância às florestas na produção agropecuária. E destacam serviços ecossistêmicos, constatados por pesquisadores, de que os ecossistemas florestais potencializam a produção agrícola (FOLI et al., 2014), por meio da regulação da água, formação do solo, proteção, circulação de nutrientes, conservação da biodiversidade, estabilidade do agroecossistema, controle de pragas e polinização. Esses serviços ligados à produção agrícola contribuem para a segurança alimentar (HLPE, 2017). O papel das florestas no ciclo hidrológico (ELLISON et al., 2017) inclui o fornecimento de matéria orgânica (KIMBLE et al., 2007), adubo verde e composto para a agricultura (SINU et al., 2012).

Acredita-se que os produtores ecológicos, no seu fazer cotidiano de manejo de sistemas agroflorestais, percebem os efeitos benéficos das interações entre as espécies, considerando os processos de sucessão ecológica, eficiência na ciclagem de nutrientes, uso de recursos naturais, presença de espécies fixadoras de nitrogênio, cobertura do solo e biodiversidade. E pesquisas apontam que as árvores, em sistemas agroflorestais, aumentam significativamente a produtividade das culturas agrícolas, pois as florestas abrigam espécies da fauna cruciais para a polinização das culturas alimentares (ROUBIK, 1995), e a diversidade de polinizadores pode aumentar, significativamente, a intensidade da polinização (GARIBALDI et al., 2016). 
Provavelmente, os agricultores citam os serviços de regulação porque reconhecem a dependência que possuem desses serviços, para as atividades produtivas e o bem-estar humano. Martín-López et al. (2012), em pesquisa na Península lbérica, também constataram que os serviços, mais frequentemente percebidos, são os de regulação, e que a percepção dos serviços ecossistêmicos varia em consequência da estratégia de manejo da terra.

\section{Fontes de informação e de formação sobre as florestas}

A mídia desempenha um papel importante nas relações sociais e promove a divulgação e sensibilização das pessoas para as questões ambientais (BACCEGA, 2014; CARLI et al., 2016). Ela contribui para a construção dos saberes e influencia na postura e relação do indivíduo com o ambiente (FELDMAN, 2016).

Segundo os agricultores participantes da pesquisa, os conhecimentos elaborados sobre a importância das florestas para a agropecuária são influenciados pelas fontes de informações acessadas, pela conversa com outros agricultores e pela assistência/assessoria técnica recebida.

A televisão (citada por $71,66 \%$ ) e o rádio (citado por $48,33 \%$ ) são as principais fontes de informação sobre as florestas, seguidas pela internet e materiais impressos - boletins, folhetos, jornais e revistas (Tabela 4). Porém, evidencia-se que existem diferenças entre a porcentagem de citações dessas fontes de informação entre os agricultores convencionais e os agroecológicos $\left(x^{2}=82,54 ; \mathrm{gl}=3 ; \mathrm{p}<0,05\right)$, entre os agricultores de diferentes faixas de idades $\left(x^{2}=16,36 ; \mathrm{gl}=3, \mathrm{p}<0,001\right)$ e entre aqueles com diferentes níveis de instrução $\left(x^{2}=31,18 ; \mathrm{gl}=3 ; \mathrm{p}<0,0001\right)$. A televisão é mais citada entre os produtores convencionais; já a internet e os materiais impressos, entre os agroecológicos. Também se verifica que o número de citações da televisão e do rádio decresce conforme diminui a idade e aumenta o nível de instrução dos agricultores; ao passo que aumentam as citações da Internet e dos materiais impressos, conforme diminui a idade e aumenta o nível de instrução.

Tabela 4: Fontes de informações sobre as florestas, segundo produtores rurais, residentes no Norte do RS, agrupados segundo o tipo de agricultura praticada na propriedade; sexo, idade e escolaridade (\% de citações)

\begin{tabular}{lcccccccccc}
\hline & \multicolumn{1}{c}{ Agricultura } & \multicolumn{2}{c}{ Sexo } & \multicolumn{3}{c}{ Idade } & \multicolumn{3}{c}{ Escolaridade } \\
\cline { 2 - 11 } Fontes & Conv. & Agro. & Fem & Masc & $20-39$ & $40-59$ & +60 & EF & EM & EM \\
& $\mathrm{n}=60$ & $\mathrm{n}=60$ & $\mathrm{n}=40$ & $\mathrm{n}=80$ & $\mathrm{n}=25$ & $\mathrm{n}=68$ & $\mathrm{n}=27$ & $\mathrm{n}=55$ & $\mathrm{n}=35$ & $\mathrm{n}=30$ \\
\hline TV & 85.0 & 58.3 & 65.0 & 75.0 & 72.0 & 66.2 & 85.2 & 78.2 & 71.4 & 60.0 \\
\hline Rádio & 55.0 & 41.7 & 45.0 & 50.0 & 56.0 & 41.2 & 59.3 & 58.2 & 51.4 & 26.7 \\
\hline Internet & 31.7 & 38.3 & 35.0 & 35.0 & 52.0 & 38.2 & 11.1 & 12.7 & 40.0 & 70.0 \\
\hline Materiais & \multirow{2}{*}{10.0} & 26.7 & 22.5 & 16.3 & 24.0 & 19.1 & \multirow{2}{*}{11.1} & \multirow{2}{*}{3.6} & 22.9 & 40.0 \\
impressos
\end{tabular}

Fonte: Compilado pelos autores a partir dos dados primários (2019). 
Provavelmente, a televisão é uma das principais fontes de informação sobre as florestas, por estar presente na residência de todas as famílias, por adotar uma linguagem simples e pela sua capacidade de persuasão do grande público. No Brasil, a televisão, a partir da década de 1950, ganha destaque entre a população brasileira, ocupando gradativamente o lugar de outros meios de comunicação (jornais, folhetos informativos e o rádio). Segundo o IBGE (2018), apenas 2,8\% dos brasileiros não possuem televisão pública em seus domicílios, e $77 \%$ dos brasileiros assistem televisão todos os dias (BRASIL, 2016). E, apesar da popularização e do crescente uso das mídias digitais, a televisão é a principal fonte de informação para os participantes da pesquisa.

Em 2020, pela primeira vez as redes sociais estão à frente da TV como fonte de informação para os brasileiros. São $67 \%$ os que dizem usá-las para se informar, e $66 \%$ os que citam a televisão (REUTERS INSTITUTE FOR THE STUDY OF JOURNALISM,2020). Pesquisa aponta que o brasileiro assiste televisão, em média, durante $6 \mathrm{~h} 17 \mathrm{~min}$, diariamente, sendo utilizados diversos formatos de transmissão, incluindo a TV pela internet, TV aberta, Pay-TV, websites, app de mensagens, on demand e vídeos gratuitos na internet. $\mathrm{Na}$ comparação com o resto do mundo, a diferença é muito expressiva: enquanto a média diária mundial é de $2 \mathrm{~h} 55 \mathrm{~min}$, no Brasil, ela é mais que o dobro. A América Latina, como um todo, tem tempo médio de consumo maior do que a média global (KANTAR IBOPE MEDIA, 2020).

Já a Internet é uma fonte de informação mais adotada por agricultores que residem no meio urbano: mais jovens e com maior nível de escolarização. Ela é muito atrativa, principalmente aos mais jovens, por ser muito ágil nas comunicações e informações, além de permitir uma maior interação. Porém o seu uso é mais limitado, provavelmente, em função: i) da infraestrutura (rede de transmissão, antena, aparelhos eletrônicos), que ainda não está disponível em algumas localidades rurais das $\mathrm{RN}$ e RC; ii) da capacitação da população do campo, especialmente daqueles que possuem maior idade, para lidarem com as novas tecnologias.

O estudo identificou que $97 \%$ dos participantes costumam dialogar sobre as florestas, principalmente com familiares, outros agricultores e também com profissionais da assistência técnica e dos sindicatos. $E$, em relação à frequência com que conversam sobre o tema, há diferenças entre os produtores convencionais e os agroecológicos $(x 2=46,84 ; g l=4 ; p<0,001)$; entre homens e mulheres $(x 2=27,58 ; g l=4 ; p<0,0001)$.

Os agricultores também relatam que agregaram informações sobre as florestas por meio de formações que aconteceram no formato de cursos, palestras, oficinas e dias de campo, que trataram sobre o Código Florestal; estratégias para a conservação e uso sustentável de florestas, Cadastro Ambiental Rural; estratégias de regularização ambiental das propriedades rurais. Essas formações foram promovidas por diferentes entidades, com destaque para as ONGs, Emater RS - Ascar, Secretarias Municipais de Agricultura, Empresas Privadas, Sindicatos, Senar, Núcleo de Agroeocologia 
do Alto Uruguai (NAAU) e pela Universidade Comunitária, situada na Região (URI).

Há maior participação, em formações, pelos produtores agroecológicos. Também se verifica que, conforme diminui a idade dos agricultores e aumenta o nível de escolarização dos participantes da pesquisa, há uma tendência de maior participação em processos de formação (Figura 4).

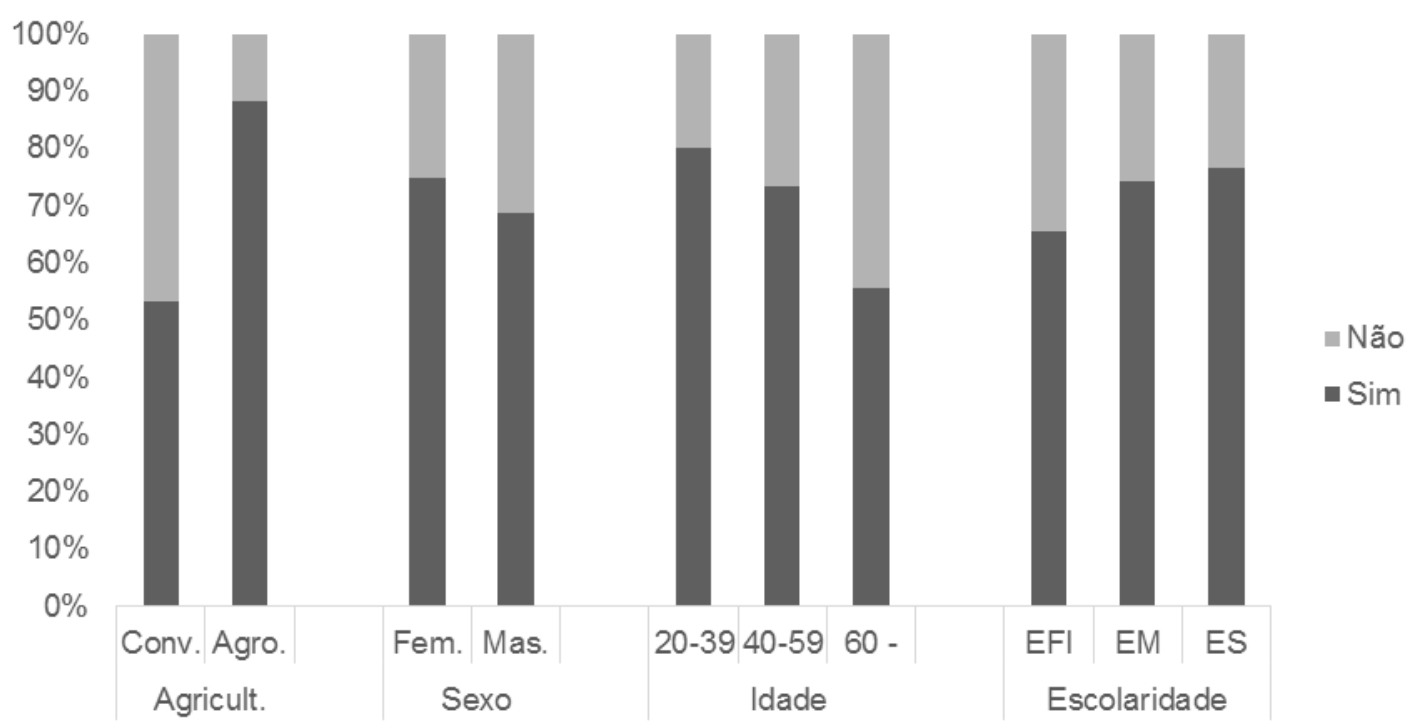

Figura 4: Participação em processos de formação sobre as florestas, pelos produtores rurais.residentes no Norte do RS, agrupados segundo o tipo de agricultura praticada na propriedade, sexo, idade e escolaridade.

Fonte: Compilado pelos autores a partir dos dados primários (2019).

A expressiva participação dos agricultores agroecológicos em formações que abrangeram o tema da pesquisa, pode ter sido influenciada pela implantação de programas governamentais na Região, atendendo ao previsto em políticas públicas voltadas à agroecologia (Lei ํo 10.831/2003 e decreto $\mathrm{n}$. . $^{-}$ 7.794/2012), que preveem ações de pesquisa, assistência técnica, gestão ambiental, formação profissional (BRASIL, 2003; BRASIL, 2012), e por meio de programas de incentivo à produção e à comercialização dos produtos agroecológicos - Programa de Aquisição de Alimentos (PAA); Programa Nacional de Alimentação Escolar (PNAE); PGPM, Política de Garantia de Preços Mínimos (PGPM); Assistência Técnica e Extensão Rural (ATER). Na Região, a implantação desses Programas contou com o apoio da EMATER RS - Ascar e das ONGs.

Os agricultores (60 agroecológicos e 42 convencionais) também afirmam receber assistência técnica/assessoria, para o planejamento e regularização ambiental e para conservação e uso sustentável das florestas, de entidades governamentais (EMATER RS - Ascar e Secretaria Municipal da Agricultura), 
ONGs e Empresas (Tabela 5). Convém destacar que, em algumas situações mais de uma entidade presta assistência/assessoria às propriedades.

Tabela 5: Entidades que prestam assessoria/assistência técnica aos agricultores residentes no Norte do RS, Sul do Brasil, agrupados segundo tipo de agricultura praticada, sexo; idade e escolaridade dos participantes (\% de citações).

\begin{tabular}{lcccccccccc}
\hline & \multicolumn{1}{c}{ Agricultura } & \multicolumn{2}{c}{ Sexo } & \multicolumn{3}{c}{ Idade } & \multicolumn{3}{c}{ Escolaridade } \\
\cline { 2 - 11 } Entidades & $\begin{array}{l}\text { Conv. } \\
\mathrm{n}=60\end{array}$ & $\begin{array}{c}\text { Agro. } \\
\mathrm{n}=60\end{array}$ & $\begin{array}{c}\text { Fem } \\
\mathrm{n}=40\end{array}$ & $\begin{array}{c}\text { Masc } \\
\mathrm{n}=80\end{array}$ & $\begin{array}{c}20-39 \\
\mathrm{n}=25\end{array}$ & $\begin{array}{c}40-59 \\
\mathrm{n}=68\end{array}$ & $\begin{array}{c}+60 \\
\mathrm{n}=27\end{array}$ & $\begin{array}{c}\mathrm{EF} \\
\mathrm{n}=55\end{array}$ & $\begin{array}{c}\text { EM } \\
\mathrm{n}=35\end{array}$ & $\begin{array}{c}\mathrm{EM} \\
\mathrm{n}=30\end{array}$ \\
\hline $\begin{array}{l}\text { Emater/ } \\
\text { Prefeitura }\end{array}$ & 13.3 & 56.7 & 40.0 & 32.5 & 84.0 & 23.5 & 18.5 & 32.7 & 42.9 & 30.0 \\
\hline Empresas & 56.7 & 6.7 & 17.5 & 38.8 & 20.0 & 35.3 & 33.3 & 29.1 & 25.7 & 43.3 \\
\hline ONGs & 0.0 & 78.3 & 52.5 & 32.5 & 48.0 & 42.7 & 22.2 & 30.9 & 54.3 & 36.7 \\
\hline
\end{tabular}

Fonte: Compilado pelos autores a partir dos dados primários (2019).

Há diferença entre as entidades que prestam assistência aos produtores agroecológicos e aos convencionais $\left(x^{2}=136.65 ; g l=3 ; p<0,0001\right)$ : as ONGs (com destaque para o Centro de Promoção e Apoio à Agroecologia - CAPA, o Centro de Tecnologias Alternativas Populares - CETAP, Rede Ecovida de Agroecologia) e a EMATER RS/Ascar orientam os produtores agroecológicos, em questões relativas à regularização ambiental das propriedades, estratégias de conservação e uso sustentável, com ênfase nos sistemas agroflorestais. As ONGs atuam, atendendo aos princípios da agroecologia e da cooperação; desenvolvem experiências de produção, beneficiamento, industrialização e comercialização; de formação e capacitação; de saúde comunitária, contribuindo para que o meio rural possa ser um espaço de vida saudável que garanta a sustentabilidade socioeconômica das propriedades. Já os agricultores convencionais são assessorados por empresas privadas, com vistas à regularização ambiental de suas propriedades, atendendo ao previsto na legislação.

Sintetizando, é possível afirmar que os agricultores participantes da pesquisa, com destaque aos agroecológicos, possuem uma compreensão mais ampla dos serviços ecossistêmicos, prestados pelas florestas à agropecuária, em função das fontes de informação acessadas e da assistência/assessoria às propriedades pelas ONGs e por entidades governamentais.

\section{Considerações finais}

O estudo evidenciou que o tipo de agricultura praticada é o principal fator que interfere nas percepções dos agricultores, residentes no Norte do Rio Grande do Sul, Brasil, sobre a importância das florestas para as atividades agropecuárias. Os agricultores agroecológicos possuem uma percepção mais ampla dos serviços gerados pelas florestas, quando comparados aos agricultores convencionais. Reconheceram e destacaram um grande número 
de serviços prestados pelas florestas e demostraram uma maior preocupação com a conservação.

É muito provável que as fontes de informações acessadas, o diálogo frequente sobre o tema e a participação em processos de formação continuada tenham contribuído para ampliar as percepções dos produtores ecológicos sobre a importância das florestas.

A conservação das florestas depende da compreensão dos agricultores em relação à sua importância de forma mais ampla, mas também da importância das florestas para as atividades produtivas rurais. E conhecer as suas percepções e as fontes de informação que orientem as suas ações, é um passo para a discussão, adoção, ampliação e/ou redirecionamentos de estratégias de proteção e intervenção, na perspectiva de amenizar os impactos gerados pelas perdas de florestas. Com base nessa compreensão é possível subsidiar o planejamento de programas educativos, destinados à agricultores voltados à conservação da biodiversidade, favorecendo propostas que considerem as necessidades locais. Também podem contribuir para a elaboração de políticas públicas, voltadas ao manejo sustentável, e para traçar planos de incentivo à preservação e recuperação das áreas florestais.

A pesquisa aponta a necessidade de investigar sobre como a temática conservação e uso sustentável das florestas vêm sendo tratados pelas entidades que prestam assistência técnica aos agricultores, bem como sobre as ideias e valores veiculados pelos meios de comunicação acessados, em especial pelos programas de televisão destinados a produtores rurais.

\section{Agradecimentos}

O presente trabalho foi realizado com apoio da Coordenação de Aperfeiçoamento de Pessoal de Nível Superior - Brasil (CAPES) - Código de Financiamento 001. Também contou com o apoio Institucional e financeiro do Programa de Pós-Graduação da Universidade Regional Integrada do Alto Uruguai e das Missões (URI).

Os autores agradecem à CAPES, à URI, às Entidades Apoiadoras e em especial, aos agricultores que contribuíram com a realização da pesquisa.

\section{Referências}

ALARCON, G. G.; FANTINI, A. C.; SALVADOR, C. H.. Benefícios Locais da Mata Atlântica: Evidências de Comunidades Rurais do Sul do Brasil. Ambient. soc., São Paulo, v.19, n.3, p. 87-112, 2016.

BACCEGA, M. A. Comunicação e consumo: educação e cidadania. In: ROCHA, R.; OROFINO, M. R. Comunicação, consumo e ação reflexiva: caminhos para a educação do futuro. Porto Alegre: Sulina, p. 189-204, 2014. 
BARDIN, L. L'analyse de contenu. Paris: PUF, 1995.

BEGON, M.; TOWSEND, C. R.; HARPER, J. L. Ecologia: de indivíduos a ecossistemas. 4. ed. Porto Alegre, Artmed, 2007.

BRASIL. Decreto $\mathrm{n}^{\circ}$ 7.794, de 20 de agosto de 2012. Institui a Política Nacional de Agroecologia e Produção Orgânica - PLANAPO, 2012.

BRASIL. MINISTÉRIO DO MEIO AMBIENTE. Brasil: 5o relatório nacional para a Convenção sobre Diversidade Biológica. Ministério do Meio Ambiente. Secretaria de Biodiversidade e Florestas. Brasília: MMA, 2016.

BRASIL. Política Nacional de Assistência Técnica e Extensão Rural PNATER. BRASIL, 2003.

CAMPANILI, M.; SCHÄFFER, W. B. Mata Atlântica: Manual de adequação ambiental, 2010.

CARLI, A. A.; COSTA, E. S. As Tecnologias da Informação e Comunicação em prol da Educação Ambiental: In: CARLI, A. A. et al. A Tecnologia em prol do meio ambiente: a partir de uma análise multidisciplinar. Rio de Janeiro: Editora Lumen Juris, 2016, pp. 203-214.

$\mathrm{CHAO}, \mathrm{S}$. et al. Forest peoples: numbers across the world. Moreton-in-Marsh: Forest Peoples Programme, 2012.

CORDEIRO, J. LP; HEINRICH, H. Cobertura vegetal atual do Rio Grande do Sul. Campos sulinos: conservação e uso sustentável da biodiversidade. Brasília: Ministério do Meio Ambiente, 2009, p.285-299.

DE SOUSA DANTAS, M., ALMEIDA, N. V., DOS SANTOS MEDEIROS, I.; DA SILVA, M. D. Diagnóstico da vegetação remanescente de Mata Atlântica e ecossistemas associados em espaços urbanos. Journal of Environmental Analysis and Progress, p.87-97, 2017.

ELLISON, D., MORRIS, C.E., LOCATELLI, B., SHEIL, D., COHEN, J., MURDIYARSO, D., GUTIERREZ, V., et al. Trees, forests and water: cool insights for a hot world. Global Environmental Change, 43: 51-61, 2017

FAO. 1948-2018. Seventy years of FAO's Global Forest Resources Assessment. Historical overview and future prospects. Food and Agriculture Organization of the United Nations. Rome, 2018. Disponível em: <http://www.fao.org/3//8227EN/i8227en.pdf>. Acesso em 12 de mai. 2020.

FAO. Global Forest Resources Assessment 2020 Key findings. Food and Agriculture Organization of the United Nations. Rome, 2018. Disponível em: $<$ http://www.fao.org/documents/card/en/c/ca8753en>. Acesso: 2 de jul. 2020;

FEIX, R. D.; LEUSIN J.S. Painel do agronegócio no Rio Grande do Sul: 2015. Porto Alegre: FEE, 2015.

FELDMAN, L. Effects of TV and Cable News Viewing on Climate Change Opinion, Knowledge, and Behavior. Oxford Research Encyclopedia of Climate $\quad 2016 . \quad$ Science. 2 Disponível: 
$<$ http://oxfordre.com/climatescience/view/10.1093/acrefore/9780190228620.001 .0001/acrefore-9780190228620-e-367>. Acesso em: 07 dez. 2018.

FINOTTI, E.; SANTOS, D. C. Análise de Ocorrência de Vendavais no Rio Grande do Sul. Ciência e Natura, Edição especial, dez, p. 518-520, 2013.

FOLI, S., REED, J. CLENDENNING, J., PETROKOFSKY, G., PADOCH, C. \& SUNDERLAND, T. To what extent does the presence of forests and trees contribute to food production in humid and dry forest landscapes? A systematic review protocol. Environmental Evidence, v.3, n. 15, 2014.

FRASER, J. A., et al. Cultural valuation and biodiversity conservation in the Upper Guinea forest, West Africa. Ecology and Society. v.21, n.3, 2016.

FRICK, J., BAUER, N., LINDERN, E.V.; HUNZIKER, M. What forest is in the light of people's perceptions and values: socio-cultural forest monitoring in Switzerland. Geogr. Helv., 73, 335-345, 2018.

GAMFELDT, Lars et al. Higher levels of multiple ecosystem services are found in forests with more tree species. Nature communications, v. 4, n. 1, p. 1-8, 2013.

GARDNER, T.A., BARLOW, J., CHAZDON, R., EWERS, R.M., HARVEY, C.A., PERES, C.A. ;SODHI N.S. Prospects for tropical forest biodiversity in a humanmodified world. Ecology Letters, n.12, p.561-582, 2009.

GARIBALDI, L. A., CARVALHEIRO, L.G., VAISSIĖRE, B.E., GEMMILLHERREN, B., HIPÓLITO, J., FREITAS, B.M., NGO, H.T., et al. Mutually beneficial pollinator diversity and crop yield outcomes in small and large farms. Science, 351: 6271, 2016.

HLPE. Sustainable forestry for food security and nutrition. A report by the High Level Panel of Experts on Food Security and Nutrition of the Committee on World Food Security. Rome, 2017. Disponível em: <http://www.fao.org/3/ai7395e.pdf>. Acesso: 12 mai. 2020.

IBGE. Censo Agropecuário. Disponível em: $<$ https://www.ibge.gov.br/estatisticas/economicas/agricultura-e-pecuaria/9827censo-agropecuario.html?=\&t=0-que-e $>$. Acesso em 2 de mar. 2019.

KANTAR IBOPE MEDIA. Inside TV Experiência, influência e as novas dimensões do vídeo. Disponível em: <https://www.kantaribopemedia.com/wpcontent/uploads/2020/03/Kantar-IBOPE-Media Inside-TV 2020-1.pdf>. Acesso em: 13 de abr. 2020

KIMBLE, J. M., RICE, C.W., REED, D., MOONEY, S., FOLLETT, R.F. ;LAL, R. (eds.) Soil carbon management: Economic, environmental and societal benefits. CRC Press, Boca Raton, USA, 2007.

MARTÍN-LÓPEZ, B. et al.(2012). Uncovering ecosystem service bundles through social preferences. PLoS One, n.7, v.6, e38970, 2012. 
MEIJAARD, E., ABRAM, N. K., WELLS, J. A., PELLIER, A. S., ANCRENAZ, M., GAVEAU, D. L., et al. People's perceptions about the importance of forests on Borneo. PloS one, v.8, n.9, e73008, 2013.

NEDEL, A. S.; SAUSEN, T. M.; SAITO, S. M. Zoneamento dos desastres naturais ocorridos no estado do Rio Grande do Sul no período 2003-2009Parte II: Granizo e Vendaval. Revista Brasileira de Meteorologia, n.27, v.2, p.119-126, 2012.

ONU. Transforming our world: the 2030 Agenda for Sustainable Development. Objetivo 15, 2015.

OUKO, C.A.; MULWA, R.; KIBUGI,R.; OWUOR, M.A.; ZAEHRINGER, J.G.; OGUGEN.O.. Community Perceptions of Ecosystem Services and the Management of Mt. Marsabit Forest in Northern Kenya. Environments, n.5, v.11, 121, 2018.

PERFECTO, I.; VANDERMEER, J. Biodiversity conservation in tropical agroecosystems. Annals of the New York Academy of Sciences, v. 1134, n. 1, p. 173-200, 2008.

QUINTAS-SORIANO, C.; BRANDT, J. S.; RUNNING, K.; BAXTER, C. V.; GIBSON, D. M.; NARDUCCI, J.; et al. Social-ecological systems influence ecosystem service perception: a Programme on Ecosystem Change and Society (PECS) analysis. Ecology and Society, n.23, v.3, 2018.

RAMALHO, M.; POLINO, C.; MASSARANI, L. Do laboratório para o horário nobre: a cobertura de ciência no principal telejornal brasileiro. JCOM, Trieste, Sissa, v. 11, n. 2, p. A02, 2012.

REUTERS INSTITUTE FOR THE STUDY OF JOURNALISM. Reuters Digital News Report. Reuters Institute: University Oxford, 2020. Disponível: https://reutersinstitute.politics.ox.ac.uk/sites/default/files/2020-

06/DNR_2020_FINAL.pdf. Acesso em: 20 out. 2020.

RIO GRANDE DO SUL. Perfil Socioeconômico-COREDE Celeiro. Governo do Estado do Rio Grande do Sul Secretaria do Planejamento, Mobilidade e Desenvolvimento Regional Departamento de Planejamento Governamental. 2105.

RIO GRANDE DO SUL. Plano estratégico de desenvolvimento regional Corede Norte - RS 2015- 2030. Porto Alegre: Edi FAPES, 2017.

ROUBIK, D. W. (ed.) 1995. Pollination of cultivated plants in the tropics. FAO Agricultural Services Bulletin 118. FAO, Rome, 1995. Disponível em: $<$ http://www.fao.org/3/a-v5040e.pdf >. Acesso em 12 mai. 2020.

SANTOS, L. et al. Políticas públicas para o comércio de produtos orgânicos no Brasil. Revista de Ciências Agrárias, v. 40, n. 2, p. 170-180, 2017.

SILVA, J. S. B. Percepção de comunidades rural e urbana sobre a importância das florestas e das unidades de conservação de Pernambuco. Recife, 2016. 
SINARE, H.; GORDON, L. J.; KAUTSKY; E. ENFORS. Assessment of ecosystem services and benefits in village landscapes-A case study from Burkina Faso. Ecosystem services, n.21, p.141-152, 2016.

SINU, P. A., KENT, S.M. \& CHANDRASHEKARA, K. Forest resource use and perception of farmers on conservation of a usufruct forest (Soppinabetta) of Western Ghats, India. Land Use Policy, v.29: p.702- 709, 2012.

SOARES-FILHO, B. et al. Cracking Brazil's forest code. Science, v. 344, n. 6182, p. 363-364, 2014.

SODHI, N. S. et al. Local people value environmental services provided by forested parks. Biodiversity and Conservation, v. 19, n. 4, p. 1175-1188, 2010.

VARJABEDIAN, R. Lei da Mata Atlântica: retrocesso ambiental. Estudos avançados, v.24, n.68, p.147-160, 2010.

ZAKRZEVSKI, S. B. B., VARGAS, C. V., \& DECIAN, V. S. Perceptions of Farmers in Northern Rio Grande do Sul on Ecosystem Services Provided by Forests. Research, Society and Development, v.9, n.5. 